\title{
Electrical discharge machining of carbon nanomaterials in air: machining characteristics and the advanced field emission applications
}

\author{
Jong Girl Ok ${ }^{1,2}$, Bo Hyun Kim ${ }^{3}$, Do Kwan Chung ${ }^{2}$, Woo Yong Sung ${ }^{2}$, \\ Seung Min Lee ${ }^{2}$, Se Won Lee ${ }^{2}$, Wal Jun Kim², Jin Woo Park ${ }^{2}$, \\ Chong Nam Chu ${ }^{2}$ and Yong Hyup Kim ${ }^{2,4}$ \\ ${ }^{1}$ Department of Mechanical Engineering, University of Michigan, 2350 Hayward St, Ann Arbor, \\ MI 48109, USA \\ ${ }^{2}$ School of Mechanical and Aerospace Engineering, Seoul National University, San 56-1, Sillim-dong, \\ Gwanak-gu, Seoul 151-742, Korea \\ ${ }^{3}$ School of Mechanical Engineering, Andong National University, 388 Songcheon-dong, Andong, \\ Kyungbuk 760-749, Korea \\ ${ }^{4}$ Institute of Advanced Aerospace Technology, Seoul National University, San 56-1, Sillim-dong, \\ Gwanak-gu, Seoul 151-742, Korea \\ E-mail: yongkim@snu.ac.kr
}

Received 26 July 2007, in final form 25 October 2007

Published 21 December 2007

Online at stacks.iop.org/JMM/18/025007

\begin{abstract}
A reliable and precise machining process, electrical discharge machining (EDM), was investigated in depth as a novel method for the engineering of carbon nanomaterials. The machining characteristics of EDM applied to carbon nanomaterials 'in air' were systematically examined using scanning electron microscopy (SEM), high-resolution transmission electron microscopy (HR-TEM), energy-dispersive x-ray spectroscopy (EDS), x-ray photoelectron spectroscopy (XPS) and Raman spectroscopy. The EDM process turned out to 'melt' carbon nanomaterials with the thermal energy generated by electrical discharge, which makes both the materially and geometrically unrestricted machining of nanomaterials possible. Since the EDM process conducted in air requires neither direct contact nor chemical agents, it protects the carbon nanomaterial workpieces against physical damage and unnecessary contamination. From this EDM method, several advanced field emission applications including 'top-down' patterning and the creative lateral comb-type triode device were derived, while our previously reported study on emission uniformity enhancement by the EDM method was also referenced. The EDM method has great potential as a clean, effective and practical way to utilize carbon nanomaterials for various uses.
\end{abstract}

(Some figures in this article are in colour only in the electronic version)

\section{Introduction}

Carbon nanomaterials such as carbon nanotubes [1] and carbon nanofibers (CNFs) [2, 3] have been intensively investigated for various nanoelectronic applications due to their superb properties, good stability and a high aspect ratio. There have been many efforts, for example, to develop flat panel displays
[4], light sources [5] and backlight units [6]. For wider uses, carbon nanomaterials frequently need to be cut, leveled, patterned and figured. However, some critical problems have emerged out of formerly suggested shaping methods. Chemical treatment [7] sometimes accompanies excessive damage and undesirable adsorbates. Laser irradiation [8] is mostly area-limited and quite costly. Instrument-based cutting 

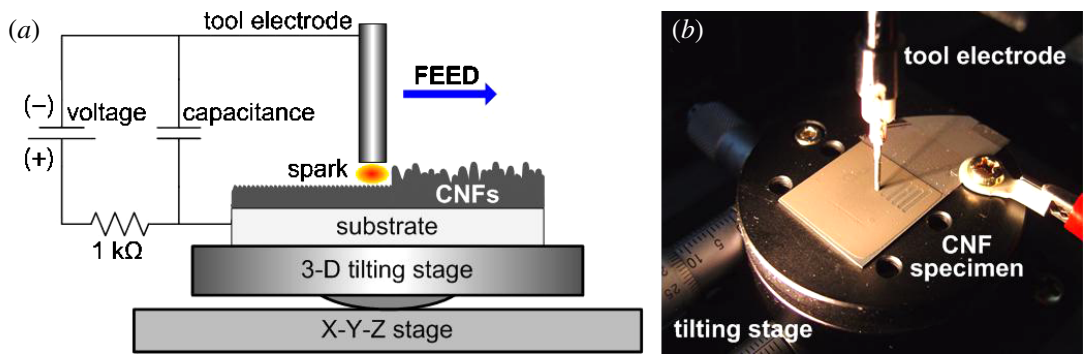

Figure 1. (a) Schematics of the EDM process applied to the CNF specimen in air, (b) a snapshot of the machining process.

using such methods as e-beam (in TEM), STM and AFM [9-12] are successfully working but are merely applicable to an individual carbon nanomaterial. Conventional 'bottomup' lithography of carbon nanomaterials for micro-nano fabrication [13] requires much labor while leaving unfavorable residues, and confines process sequences due to the inevitable exposure to a wet environment. Hence, a novel method for the reliable and extensive processing of carbon nanomaterials is strongly requested.

Here, we introduce electrical discharge machining (EDM) for the effective and multifunctional engineering of carbon nanomaterials. EDM is a robust macro/micro machining process that provides the energy for material engineering in the form of heat [14]. In EDM, a voltage difference is applied between two conductive electrodes located generally in a dielectric fluid. When the two electrodes become close enough, the dielectric fluid breaks down instantly and conducts an electrical current causing an electrical discharge spark between them. A very high temperature is then generated at the localized spots on the topmost surface of electrodes by the pinch effect of the high-frequency sparks. As a result, the electrode material is melted and, in some cases, vaporized by this concentrated thermal energy [14-16].

Since the 1950s, EDM has been broadly used for machining a variety of commercial products, especially those that are hard, complicated, curved and slender [17]. Since EDM is basically a thermal machining process and fully scalable, any conductive materials can be machined with it regardless of their mechanical/chemical properties or their machining dimensions [18]. Besides, EDM needs a very small machining force and is essentially a non-contact process, which means that the process is minutely controllable and has no limitation on the size or the shape of a tool electrode [14]. These outstanding advantages have encouraged a significant amount of research in the development of micro-EDM. A wide range of the machining availability on the workpiece materials, the tool configurations and the machining geometry makes EDM very versatile. In addition, EDM can be simply adjusted by a few electrical parameters while it requires a relatively inexpensive and compact machining system, which helps make the process reliable and practicable.

In the present study, the machining characteristics of EDM applied to CNFs in air were examined through SEM, HRTEM, EDS, XPS and Raman spectra, whose relevant features were also discussed. With the high capability of EDM and its good machining compatibility with carbon nanomaterials, various field emission devices such as the top-down patterned field emitter array and the lateral comb-type triode were investigated.

\section{Experimental details}

The CNFs used in our experiments were prepared by using the thermal chemical vapor deposition (CVD) method at $600{ }^{\circ} \mathrm{C}$ for 20 min from $\mathrm{NH}_{3} / \mathrm{C}_{2} \mathrm{H}_{2}$ gases with $\mathrm{Ni}$ catalysts deposited upon the $\mathrm{Cu}$ micro-tip structures formed on a Si substrate. A particular procedure of the CNF synthesis based on the $\mathrm{Cu}$ micro-tips has been reported elsewhere [19]. In some cases (i.e. triode fabrication), we used shorter CNFs synthesized more simply on a Ni (100 $\AA$, as catalysts)/Ti (500 $\AA$, as an electrode layer)/glass substrate.

The EDM process is normally conducted in a dielectric fluid such as kerosene or de-ionized water [20]. However, kerosene produces an array of carbonic by-products during machining [21] that could contaminate the CNF surface. Deionized water is also inadequate for processing CNFs since the machining zone by the spark is too large [14]. In the present study, the EDM process was carried out in air while the discharge energy was carefully minimized to prevent contamination or damage to the CNFs. Since CNFs are high aspect ratio structures having nano-scale diameters, they are readily machined without the use of any dielectric fluid.

We applied the EDM process to the raw surface of the CNFs in air while feeding a cylindrical tungsten carbide tool electrode, as schematically depicted in figure $1(a)$. The sizes of tool electrodes were properly chosen, with a diameter of $1 \mathrm{~mm}$ for the analysis of machining characteristics, $100 \mu \mathrm{m}$ for the patterning, and $200 \mu \mathrm{m}$ for the fabrication of a lateral comb-type triode, respectively. An RC circuit was used as the discharge circuit, which is suitable for generating highfrequency pulses using little energy. A stray capacitance was used to minimize the discharging energy. The applied voltage was $60-100 \mathrm{~V}$, which varied with the tool size. When CNFs were machined with $100 \mathrm{~V}$, stray capacitance and a $\varnothing 1 \mathrm{~mm}$ tool, the size of machining gap was about $50 \mu \mathrm{m}$. When $60 \mathrm{~V}$ was applied, however, the gap reduced to about $30 \mu \mathrm{m}$ [22]. The CNF specimen was fixed on a three-dimensional controllable tilting stage [figure $1(b)$ ] that helped keep the gap between the specimen and the tool constant over the machining area. The feed rate was set to $200 \mu \mathrm{m} \mathrm{s}^{-1}$, which was slow enough to prevent a short between the CNF specimen and the tool electrode.

For a reasonable comparison, we used a single as-grown CNF specimen for each analysis; only a partial area was 

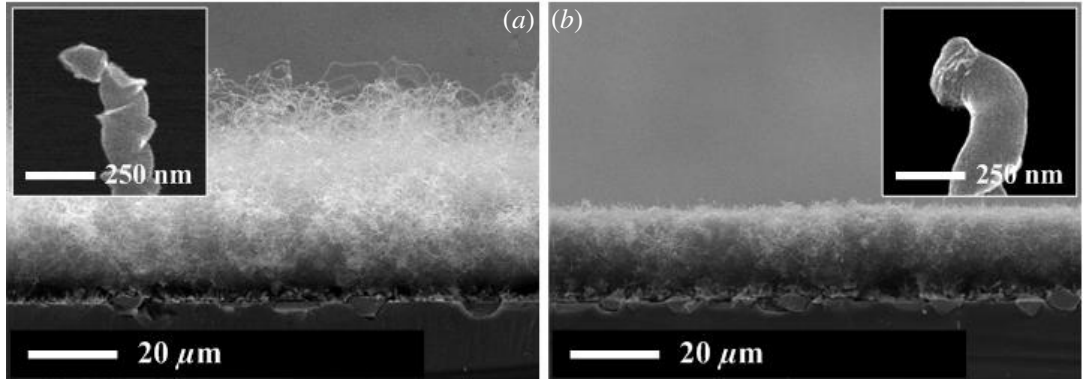

Figure 2. SEM images of CNFs: $(a)$ before and $(b)$ after EDM in air. Insets [22] are enlarged HR-SEM images of an individual CNF tip end of each case.
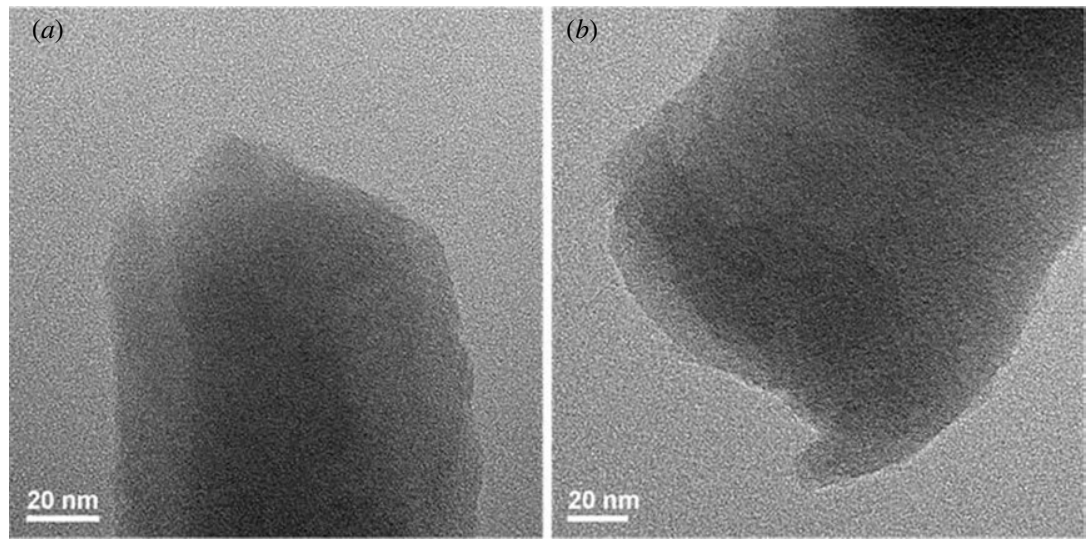

Figure 3. HR-TEM images of an individual CNF strand: (a) the non-EDMed end, $(b)$ the EDMed end.

machined while the other part remained untouched on the same specimen. Especially for the XPS and Raman spectra analyses, multilevel machining was conducted in order to verify the EDM effects on CNFs more systematically; a part of the CNF surface was machined to the depth of $10 \mu \mathrm{m}$ from the topmost surface (light machining) while the amount of $30 \mu \mathrm{m}$ depth CNFs was machined for the other part via thrice the $10 \mu \mathrm{m}$ depth machining (severe machining).

\section{Machining characteristics: analyses and discussion}

Figure 2 shows the SEM images of the CNFs before and after EDM in air. The CNFs with the as-grown height of 50$60 \mu \mathrm{m}$ in entangled configuration were effectively machined after the EDM process, having the uniform and clean surface profile, which may be more profitable for various engineering applications. Compared to the pristine CNF tip [inset of figure 2(a)], after the EDM process the CNF tip end became more blunt and appears to be melted due to the thermal energy out of the electrical discharge, as indicated in the inset of figure $2(b)$.

In order to examine the machining characteristics more closely, an HR-TEM observation in conjunction with an EDS analysis was performed. In contrast to the end of a non-EDMed CNF strand having an intact shape [figure 3(a)], the EDMed end shows a bluntly separated appearance that suggests the ends were not 'cut' but 'melted' and partly vaporized during the EDM process conducted in air. Besides, as shown in

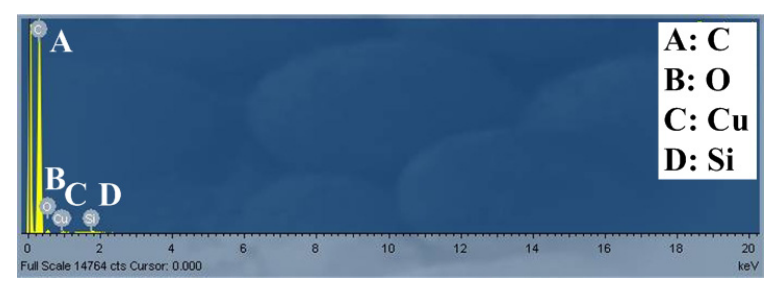

Figure 4. EDS spectra acquired from the EDMed CNF surface. The $\mathrm{C}, \mathrm{O}, \mathrm{Cu}$ and $\mathrm{Si}$ peak came out of CNFs, slight oxidation (as discussed below), $\mathrm{Cu}$ micro-tip structures and a Si substrate, respectively.

figure 4, no measurable residue of an electrode tool such as W or Co was detected in the CNF film after EDM, which ensures that the contaminative material transfer from the tool electrode to the CNF specimen did not occur. Checking the SEM and HR-TEM images along with the EDS spectra, undesirable residues as well as mechanical damages were not observed since any subsidiary chemicals and physical contacts were not required in EDM in air.

The edge of a tool electrode end was additionally inspected to clarify the driving force of EDM applied to carbon nanomaterials in air. In the normal EDM process, micro-craters are formed, undergoing the procedure wherein the tool material is instantly melted and vaporized and is shortly resolidified by the heat out of the electrical discharge in a moment [23]. Figure 5(b) evidently discloses that the same phenomenon occurs when EDM is applied to carbon nanomaterials in air as well as ordinary bulk workpiece materials in a liquid medium, which proves that the EDM 

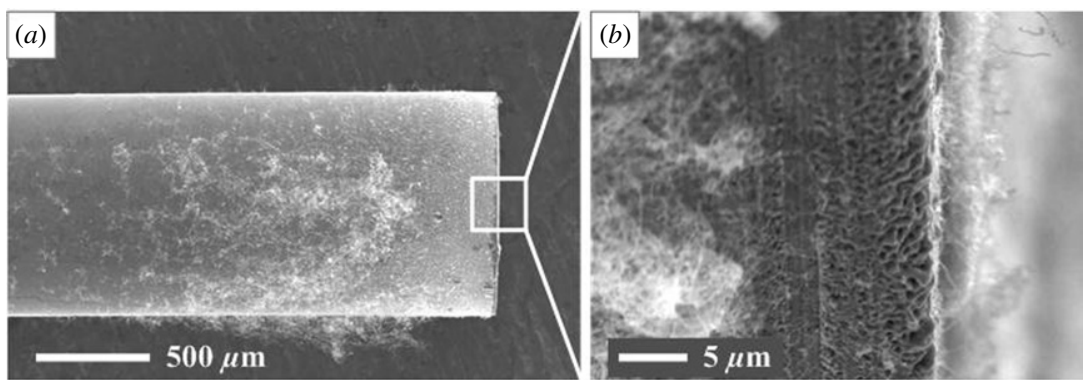

Figure 5. SEM images of the tool electrode tip after the EDM process [22]: $(a)$ an overview, $(b)$ an enlarged view of the tip edge where numerous heat-driven micro-craters were formed.

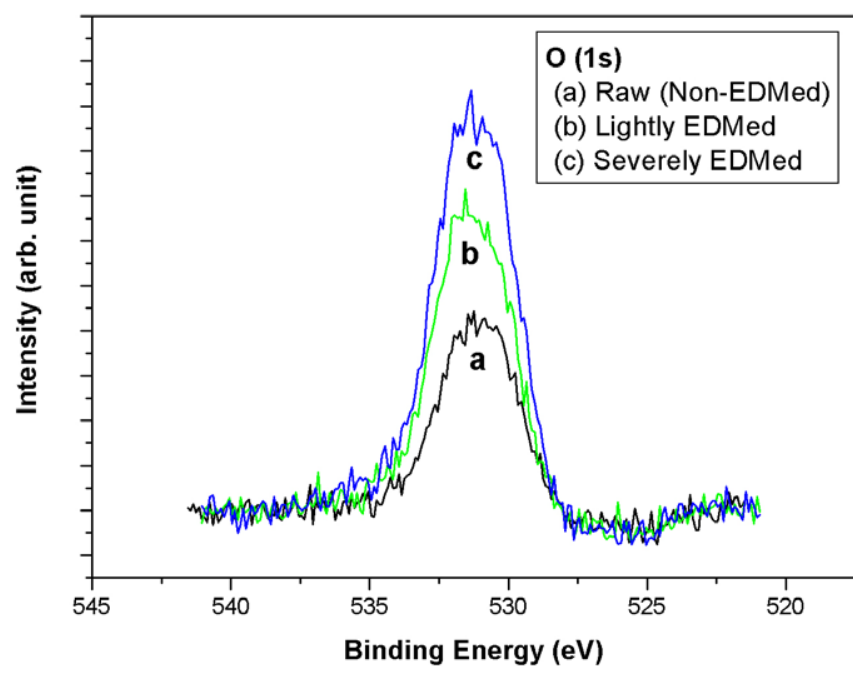

Figure 6. XPS analysis plot for $\mathrm{O}(1 \mathrm{~s})$ of the three cases: the $\mathrm{O} 1 \mathrm{~s}$ peak of $(a)$ raw, $(b)$ lightly EDMed and $(c)$ severely EDMed CNFs, respectively.

process readily engineers carbon nanomaterials in air through the use of thermal energy.

As examined in figure 5(a), some debris are usually generated and subsequently attached to the tool electrode during the EDM process with higher capacitance [22]. However, debris were successfully minimized when stray capacitance was used, and no adverse effect was found during the machining of carbon nanomaterials.

An XPS analysis was carried out to survey the oxidizing degrees since our EDM experiments were conducted in air without the support of dielectric fluids. In addition, a Raman spectra analysis was performed to check whether the crystallinity of CNFs was changed by the heat since the EDM process is conducted under very high temperatures. For a more methodical analysis, we divided the area of a single CNF specimen into three regions: raw, lightly EDMed and severely EDMed ones, as described in the experimental details. As illustrated in figure 6 , slight oxidation was entailed by the 'atmospheric' EDM process: the more EDMed, the more oxidized. The results of the XPS analysis indicate that $\mathrm{O}_{2}$ forms surface dipoles such as $\mathrm{C}-\mathrm{O}$ covalent bonds on the CNF surface [24]. Accordingly to some extent, the crystalline properties somewhat decreased, as observed in Raman spectra (figure 7). However, the change was not fatal in the respect

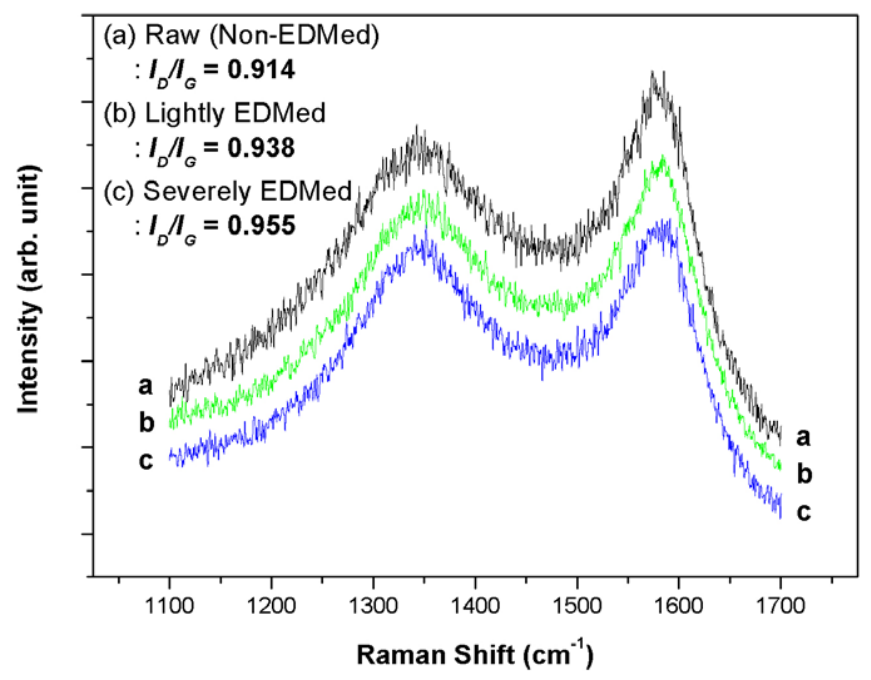

Figure 7. Raman spectra of the three cases: $(a)$ raw, (b) lightly EDMed and $(c)$ severely EDMed CNFs, respectively.

that the decrease of crystalline quality was quite faint. This demonstrates that, overall, carbon nanomaterials can be safe from thermal damage during EDM engineering in air since the discharge energy used in EDM is so small, localized and instantly applied.

In brief, carbon nanomaterials can be readily and efficiently engineered by the EDM process in air without contamination, damage and deterioration.

\section{Advanced field emission applications: the top-down patterned emitter array and lateral comb-type triode structure}

In our former research, EDM was tactfully applied to enhance the field emission uniformity of carbon nanofiber emitters through vertical leveling [25]. Here we introduce EDM to the further advancement of carbon nanomaterial-based field emission applications via the smart patterning and the handy fabrication of a lateral comb-type triode structure.

\subsection{The top-down patterning of carbon nanomaterial emitters by EDM in air}

For the purpose of attaining better practicability and higher performance, a considerable amount of research effort has 

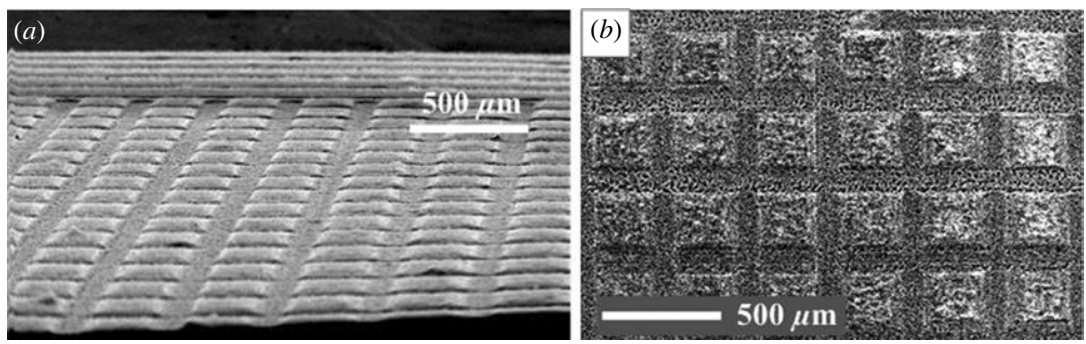

Figure 8. SEM images of the EDM-patterned CNF films in the pitch of $350 \mu \mathrm{m}$ : (a) tilted view, (b) top view.

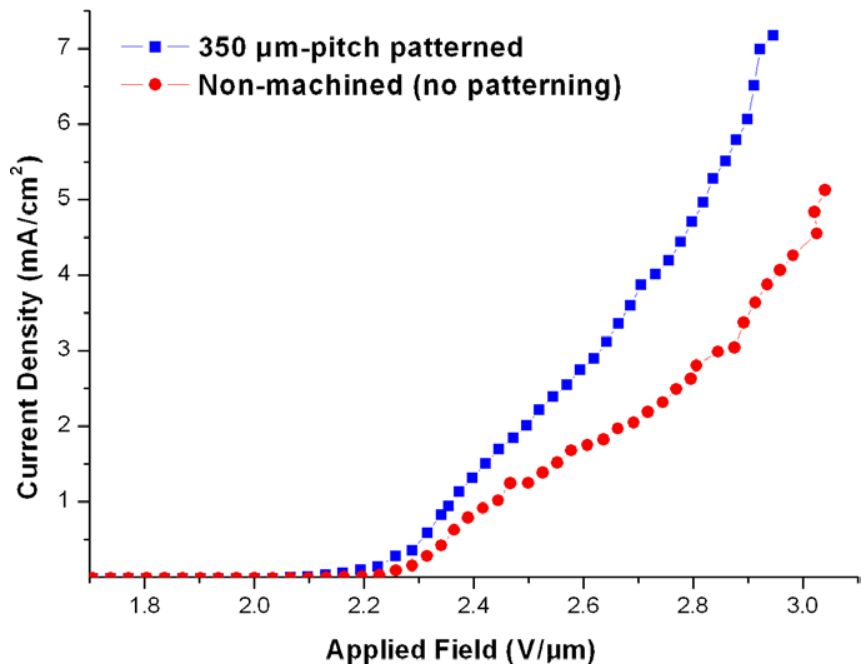

Figure 9. $I-V$ curves of the field emission: (a) the non-patterned CNF film (circles), (b) the $350 \mu$ m pitch-patterned CNF film (squares).

endeavored to develop the methods to pattern the carbon nanomaterials, such as general photolithography [13, 26], e-beam lithography [26, 27], nanoimprint lithography [28], laser-assisted patterning [29, 30], mechanical stamping [31] and dip-coating [32]. However, several problems have arisen, which include unfavorable residues, dimensional limitation, physical or chemical damage and laboriousness.

In the current study, a facile 'top-down' patterning of the CNF emitters is presented by capitalizing the EDM process in air to achieve higher field emission performance without problematic obstacles. A $\varnothing 100 \mu \mathrm{m}$ electrode tool was used in patterning by feeding in the $x-y$ directions with a specific pitch to form square-shaped patterns.

EDM was fully capable of patterning carbon nanomaterial films, as is apparent in figure 8 . The square patterns were well formed precisely in the specified dimensions. The EDM patterning method is simple and area-unrestricted, and requires neither the confinement of process sequences nor the ponderous apparatus. Also, our patterning method is free from any undesirable residues and unnecessary damage since EDM in air needs no chemical intermediates and is totally non-contact.

The field emission performance of the two cases, one without any machining or patterning at all and the other patterned in the pitch of $350 \mu \mathrm{m}$, was comparatively investigated in a vacuum chamber maintained at a base pressure of $4 \times 10^{-6}$ torr with a diode-type configuration. CNF emitters and a phosphor-coated indium-tin oxide (ITO) glass were used as the cathode and the anode, respectively. The distance between the CNF emitters and the anode was $610 \mu \mathrm{m}$. The emission current density was evaluated by averaging the measured current over the active area of $0.2827 \mathrm{~cm}^{2}$.

Figure 9 indicates the field emission $I-V$ characteristics with and without EDM patterning. The field emission current

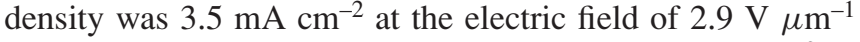
before EDM patterning, while it doubled $\left(7.0 \mathrm{~mA} \mathrm{~cm}{ }^{-2}\right.$ at the same field) after the CNF emitters were patterned by EDM. The turn-on fields (i.e. the field at the current density of $10 \mu \mathrm{A} \mathrm{cm}^{-2}$ ) of the raw and the EDM-patterned emitters were

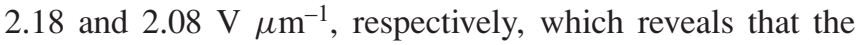
electric field concentration became more efficient after EDM patterning.

The performance improvement of the patterned emitters can be explained on the basis of two theoretical concepts: the screening effect $[33,34]$ and the edge effect $[35,36]$. When the field emitters lie too much adjacent to one another, an electrostatic screening effect is provoked, negatively affecting the field emission performance. The edge effect points out that the electrons can be emitted more effectively at the edges of the cathode due to the concentration of the electric field. The patterning treatment is able to supply the densely packed emitters with an appropriate interspace so that the screening effect could be reduced. Moreover, since EDM formed orderly patterns having valid edges on the flat CNF film, the edge effect was beneficially induced.

\subsection{The fabrication of the lateral comb-type triode structure by EDM in air}

Another ingenious field emission device is demonstrated by applying EDM to carbon nanomaterial emitters. Recently, many kinds of triode structures for field emission have been presented for high quality performance and reliability [35, 37-40]. In this paper, we propose a competent lateral-gate type triode device creatively derived from the EDM method.

Figure 10(a) illustrates the concept of the lateral combtype triode fabrication by using EDM. The comb-shaped line was chosen to divide the CNF film into two lateral electrodes since it provides a long and uniform gap per unit area between the cathode and the gate, which encourages more effective interactions. Also the comb line is easy to process. It can be completed by the single line processing of EDM, which electrically separates an electrode into two parts. One part is 
(a)

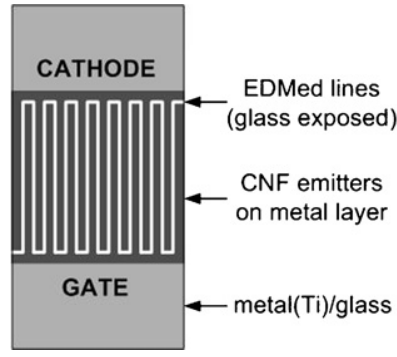

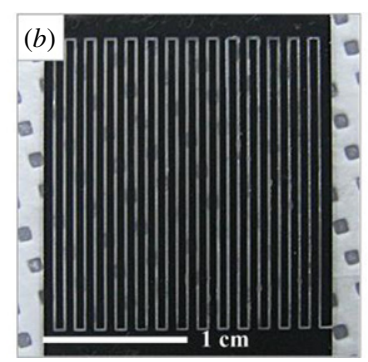

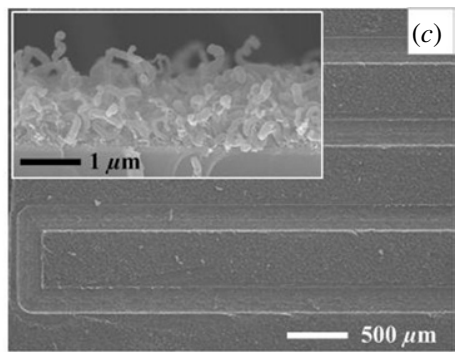

Figure 10. A lateral triode field emitter structure fabricated via the EDM method: $(a)$ schematic top view of the lateral cathode/gate plate, $(b)$ photograph of the fabricated device, $(c)$ SEM images of the fabricated device. The inset of $(c)$ shows as-grown CNF emitters used in the device, having an average height of $2 \mu \mathrm{m}$.
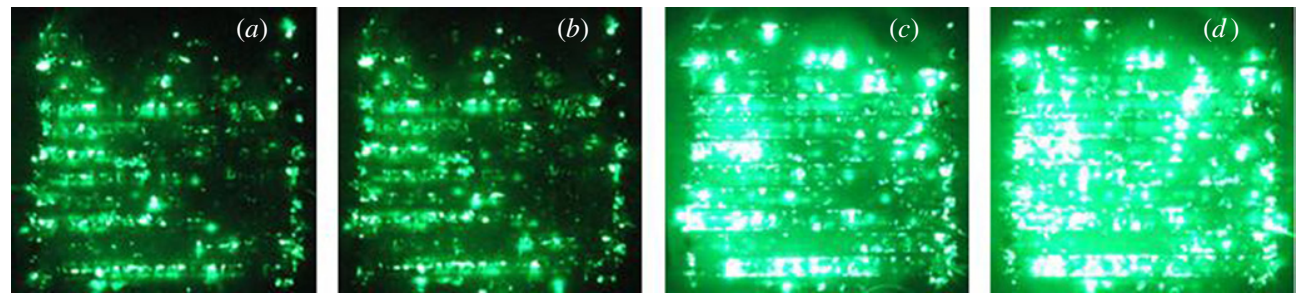

Figure 11. Emission images of a lateral comb-type triode device fabricated by the EDM method: $(a)$ diode driving, $(b)-(d)$ triode driving with the pulsed gate voltage of 100, 200 and $300 \mathrm{~V}$, respectively. For all cases, the frequency and the duty of the pulsed gate voltage were $60 \mathrm{~Hz}$ and $50 \%$, respectively, and the anode voltage was fixed to dc $2500 \mathrm{~V}$.

supplied by a pulse-type electrical power (a gate), while the other is grounded (a cathode). As the power is supplied into two parts alternately, each part works as a gate and a cathode alternately, too.

After the direct synthesis of CNF emitters on a Ni/Tideposited glass substrate, EDM using a $\varnothing 200 \mu \mathrm{m}$ tool was applied in air to machine the comb-shaped lines, removing both the CNFs and the metal layers. As clearly shown in figure $10(b)$, the lateral triode field emitter structure was capably fabricated by the EDM method. Figure $10(c)$ shows the SEM image of a part of the EDM-derived triode device, where the cathode area and the gate area were distinctly separated by EDM. In general, as the width of each comb line decreases, more uniform emission can be obtained since the resolution increases, whereas it becomes harder to fabricate. Here we set the line width to $500 \mu \mathrm{m}$, and the total machining area was $2 \times 2 \mathrm{~cm}^{2}$.

The field emission characteristics were investigated with a lateral triode configuration in a vacuum chamber. The distance between the cathode/gate plate and the phosphor/ITO-coated anode plate was $1210 \mu \mathrm{m}$, and the actual emitting area was $1.5 \times 1.5 \mathrm{~cm}^{2}$.

Figure 11 displays the emission images of each case at the same anode-to-cathode potential. Without the application of the gate voltage (i.e. diode driving), the emitting aspect was quite dimmed, and many of the emitters were still not working since the electrons could not turn on with the anode potential only [figure $11(a)$ ]. There was almost no difference when the gate voltage of $100 \mathrm{~V}$ was initially applied [figure 11(b)], which reveals that the gate did not sufficiently perform its role yet. However, when the gate voltage increased to $200 \mathrm{~V}$ [figure $11(c)$ ] and higher to $300 \mathrm{~V}$ [figure $11(d)$ ], the emitting brightness was much more enhanced with a better distribution of emission sites. This indicates that the gate potential effectively promotes the turn-on of more electrons from the cathode and accelerates their action [37, 38]. Since the electric field strength is concentrated at the edges of the cathode electrode due to the edge effect $[35,36]$, electrons start to emit first from the edges rather than the central area of the cathode electrode. Therefore, an increase in the gate voltage surrounding the cathode enhances the electric field strength around the cathode electrode, leading to the more effective electron emission from its edges [37]. As a consequence, the modulation of the gate voltage, rather than that of the anode, has more influence on the electric field at the emitters owing to the proximity of the gate to the cathode [41]. Our device is not fully optimized at this moment, and further investigations including quantitative and parametric researches are now in progress.

\section{Conclusions}

In summary, we examined the machining characteristics of EDM applied to carbon nanomaterials in air, and introduced EDM to the versatile utilization of carbon nanomaterials. Through systematic analyses, EDM was proved to process carbon nanomaterials readily in air with thermal energy. EDM as a simple and effective method for carbon nanomaterial machining has various advantages such as configurative flexibility, broad applicability and good reproducibility, and is free from contamination or damage as well. Accordingly, several advanced field emission applications derived from the EDM method were presented and their characteristics were investigated, which include the patterned field emitter array and the lateral comb-type triode device. It is promising that our original approach can design and commercialize diverse 
practicable applications such as large-scale, reproducible and high-quality field emission devices.

\section{Acknowledgments}

This work was supported by a grant (code no. 07K150102430) from the Center for Nanostructured Materials Technology under the 21st Century Frontier R\&D Programs of the Ministry of Science and Technology, and the Brain Korea 21 Project in 2007, Korea.

\section{References}

[1] Iijima S 1991 Helical microtubules of graphitic carbon Nature 354 56-8

[2] Chen Y, Patel S, Ye Y, Shaw D T and Guo L 1998 Field emission from aligned high-density graphitic nanofibers Appl. Phys. Lett. 73 2119-21

[3] Tanaka K, Yoshimura M, Okamoto A and Ueda K 2005 Synthesis of carbon nanocoils by two-step growth method using microwave plasma-enhanced chemical vapor deposition Japan. J. Appl. Phys. 44 L1158-60

[4] de Heer W A, Chatelain A and Ugarte D 1995 A carbon nanotube field-emission electron source Science 270 1179-80

[5] Saito Y and Uemura S 2000 Field emission from carbon nanotubes and its application to electron sources Carbon 38 169-82

[6] Lee S, Im W B, Kang J H and Jeon D Y 2005 Low temperature burnable carbon nanotube paste component for carbon nanotube field emitter backlight unit $J$. Vac. Sci. Technol. B $23745-8$

[7] Li J and Zhang Y 2006 Cutting of multi-walled carbon nanotubes Appl. Surf. Sci. 252 2944-8

[8] Bai X, Li D, Du D, Zhang H, Chen L and Liang J 2004 Laser irradiation for purification of aligned carbon nanotube films Carbon 42 2125-7

[9] Yuzvinsky T D, Fennimore A M, Mickelson W, Esquivias C and Zettl A 2005 Precision cutting of nanotubes with a low-energy electron beam Appl. Phys. Lett. 86 053109(1-3)

[10] Rubio A, Apell S P, Venema L C and Dekker C 2000 A mechanism for cutting carbon nanotubes with a scanning tunneling microscope Eur. Phys. J. B 17 301-8

[11] Stepanek I, Maurin G, Bernier P, Gavillet J, Loiseau A, Edwards R and Jaschinski O 2000 Nano-mechanical cutting and opening of single wall carbon nanotubes Chem. Phys. Lett. 331 125-31

[12] Park J Y, Yaish Y, Brink M, Rosenblatt S and McEuen P L 2002 Electrical cutting and nicking of carbon nanotubes using an atomic force microscope Appl. Phys. Lett. 80 4446-8

[13] Lu S and Panchapakesan B 2006 Nanotube micro-optomechanical actuators Appl. Phys. Lett. 88 253107(1-3)

[14] Masuzawa T 2000 State of the art of micromachining Ann. CIRP 49/2 473-88

[15] Wong Y S, Rahman M, Lim H S, Han H and Ravi N 2003 Investigation of micro-EDM material removal characteristics using single RC-pulse discharges J. Mater. Process. Technol. 140 303-7

[16] McGeough J A 1988 Advanced Methods of Machining (London: Chapman and Hall)

[17] Livshits A L 1960 Electro-erosion Machining of Metals (London: Butterworth)

[18] Allen D M and McGeough J A 2002 Micromachining of Engineering Materials (New York: Dekker)
[19] Sung W Y, Ok J G, Kim W J, Lee S M, Yeon S C, Lee H Y and Kim Y H 2007 Synthesis and field emission characteristics of carbon nanocoils with a high aspect ratio supported by copper micro-tips Nanotechnology 18 245603(1-5)

[20] Chung D K, Kim B H and Chu C N 2007 Micro electrical discharge milling using deionized water as a dielectric fluid J. Micromech. Microeng. 17 867-74

[21] Kim B H, Park B J and Chu C N 2006 Fabrication of multiple electrodes by reverse EDM and their application in micro ECM J. Micromech. Microeng. 16 843-50

[22] Kim B H, Ok J G, Kim Y H and Chu C N 2007 Ann. CIRP 56/1 233-6

[23] Jilani S T and Pandey P C 1986 Plasma channel growth and the resolidified layer in EDM Precis. Eng. 8 104-10

[24] Dean K A and Chalamala B R 1999 The environmental stability of field emission from single-walled carbon nanotubes Appl. Phys. Lett. 75 3017-9

[25] Ok J G, Kim B H, Sung W Y, Chu C N and Kim Y H 2007 Uniformity enhancement of carbon nanofiber emitters via electrical discharge machining Appl. Phys. Lett. $90033117(1-3)$

[26] Behnam A, Choi Y, Noriega L, Wu Z, Kravchenko I, Rinzler A G and Ural A 2007 Nanolithographic patterning of transparent, conductive single-walled carbon nanotube films by inductively coupled plasma reactive ion etching $J$. Vac. Sci. Technol. B 25 348-54

[27] Teo K B K, Chhowalla M, Amaratunga G A J, Milne W I, Hasko D C, Pirio G, Legagneux P, Wyczisk F and Pribat D 2001 Uniform patterned growth of carbon nanotubes without surface carbon Appl. Phys. Lett. 79 1534-6

[28] Vieira S M C, Teo K B K, Milne W I, Groening O, Gangloff L, Minoux E and Legagneux P 2006 Investigation of field emission properties of carbon nanotube arrays defined using nanoimprint lithography Appl. Phys. Lett. 89 022111(1-3)

[29] Sohn J I, Lee S, Song Y H, Choi S Y, Cho K I and Nam K S 2001 Patterned selective growth of carbon nanotubes and large field emission from vertically well-aligned carbon nanotube field emitter arrays Appl. Phys. Lett. 78 901-3

[30] Cheong F C, Lim K Y, Sow C H, Lin J and Ong C K 2003 Large area patterned arrays of aligned carbon nanotubes via laser trimming Nanotechnology 14 433-7

[31] Liu P, Liu L, Wei Y, Sheng L and Fan S 2006 Enhanced field emission from imprinted carbon nanotube arrays Appl. Phys. Lett. $89073101(1-3)$

[32] Huang J, Fan R, Connor S and Yang P 2007 One-step patterning of aligned nanowire arrays by programmed dip coating Angew. Chem. Int. Ed. 46 2414-7

[33] Nilsson L, Groening O, Emmenegger C, Kuettel O, Schaller E, Schlapbach L, Kind H, Bonard J-M and Kern K 2000 Scanning field emission from patterned carbon nanotube films Appl. Phys. Lett. 76 2071-3

[34] Suh J S, Jeong K S, Lee J S and Han I 2002 Study of the field-screening effect of highly ordered carbon nanotube arrays Appl. Phys. Lett. 80 2392-4

[35] Choi W B, Jin Y W, Kim H Y, Lee S J, Yun M J, Kang J H, Choi Y S, Park N S, Lee N S and Kim J M 2001 Electrophoresis deposition of carbon nanotubes for triode-type field emission display Appl. Phys. Lett. 78 1547-9

[36] Fujii S, Honda S-I, Machida H, Kawai H, Ishida K, Katayama M, Furuta H, Hirao T and Oura K 2007 Efficient field emission from an individual aligned carbon nanotube bundle enhanced by edge effect Appl. Phys. Lett. $90153108(1-3)$

[37] Choi Y S et al 2001 An under-gate triode structure field emission display with carbon nanotube emitters Diamond Relat. Mater. 10 1705-8

[38] Han I T, Kim H J, Park Y J, Lee N S, Jang J E, Kim J W, Jung $\mathrm{J}$ E and Kim J M 2002 Fabrication and characterization of 
gated field emitter arrays with self-aligned carbon nanotubes grown by chemical vapor deposition Appl. Phys. Lett. 81 2070-2

[39] Lee J H, Lee M B, Hahm S H, Lee J H, Seo H I, Kwon D H, Kim J S and Choi K M 2003 Lateral field emitter arrays with high emission currents and wide operation region by high field activation J. Vac. Sci. Technol. B 21 506-10
[40] Jung Y J, Son G H, Park J H, Kim Y W, Berdinsky A S, Yoo J B and Park C Y 2005 Fabrication and properties of under-gated triode with CNT emitter for flat lamp Diamond Relat. Mater. 14 2109-12

[41] Wong Y M, Kang W P, Davidson J L, Hofmeister W, Wei S and Huang J H 2005 Device characterization of carbon nanotubes field emitters in diode and triode configurations Diamond Relat. Mater. 14 697-703 